\title{
THE SIZE EFFECT IN MECHANICAL PROPERTIES OF BORON NITRIDE NANOTUBE UNDER TENSION
}

\author{
Nguyen Danh Truong \\ School of Mechanical Engineering, Hanoi University of Science and Technology \\ No. 1 Dai Co Viet Road, Hanoi, Vietnam. \\ "Email: truong.nguyendanh@hust.edu.vn
}

Received: 1 April 2017; Accepted for publication: 15 May 2017

\begin{abstract}
This work aimed at investigating the mechanical properties of boron nitride nanotubes (BN-NTs) under uniaxial tension using atomic finite element method with Tersoff potential. The zigzag and armchair nanotubes with different length and diameter are considered for researching effect on mechanical behavior of BN-NTs. It is found that Young's modulus of BN-NTs is independent of the tubular length, but slightly increases when the diameter goes rise. At the given strain, axial stress in the armchair tubes is higher than that in the zigzag ones. This paper will provide useful information about the mechanical properties and failure behaviors of $\mathrm{BN}$ NTs for their applications.
\end{abstract}

Keywords: atomistic simulation; tension; Boron nitride nanotubes, mechanical properties.

\section{INTRODUCTION}

In the past decades, the discovery of the superior mechanical and other physical properties in tubular nanomaterials such as carbon nanotubes (CNTs) have triggered great interest in their possible engineering applications, e.g. nanomechanical resonators [1 - 3], reinforcement for the nanocomposites [4 - 6]. Similar to that of CNTs [7], boron nitride nanotubes (BN-NTs) [8] can be geometrically formed by rolling up a hexagonal boron nitride (BN) layer in which alternating $\mathrm{B}$ and $\mathrm{N}$ atoms entirely substitute for $\mathrm{C}$ atoms as shown in Figure 1. Various techniques have been used to synthesize BN-NTs, including arc-discharge [8 - 10], chemical vapor deposition [11 - 14], laser ablation [15 - 17], etc. BN-NTs exhibit good mechanical properties with high elastic modulus of $\sim 0.5-1 \mathrm{TPa}$ [18 - 12]. They possess distinguishable chemical and thermal stability with high oxidation resistance up to $900^{\circ} \mathrm{C}$ in air [22], wide band-gaps independent of tube structures [23, 24], excellent thermal conductivity [25]. Potential applications of BN-NTs include nanofillers in polymeric [26] and metallic [27] composites, optoelectronic fields [28], radiation shielding in space vehicles [29], etc. Thus, the understanding of mechanical properties of BN-NTs is required to make be successfully employed them in the aforementioned applications. Suryavanshi et al. [18] determined the elastic modulus of BN-NTs using electricfield-induced resonance method. The measurements on 18 individual BN-NTs were reported 
with an average elastic modulus of $722 \mathrm{GPa}$. Anoop Krishnan and Ghosh studied the failure behavior of BNNTs under tension [30] and they also reported a study on the effect of chirality on elastic properties of BNNTs under uniaxial and torsional loading [31] using molecular dynamics simulations. To the best of the author' knowledge, there have not been any reported study on the effect of length and diameter on the mechanics properties of BN-NTs using atomic finite element method (AFEM).

In this work, the mechanical properties of BN-NTs under tension are investigated through AFEM. The stress-strain curve, Young's modulus, Poisson's ratio and failure behavior are studied. Effects of tube length and diameter are considered.
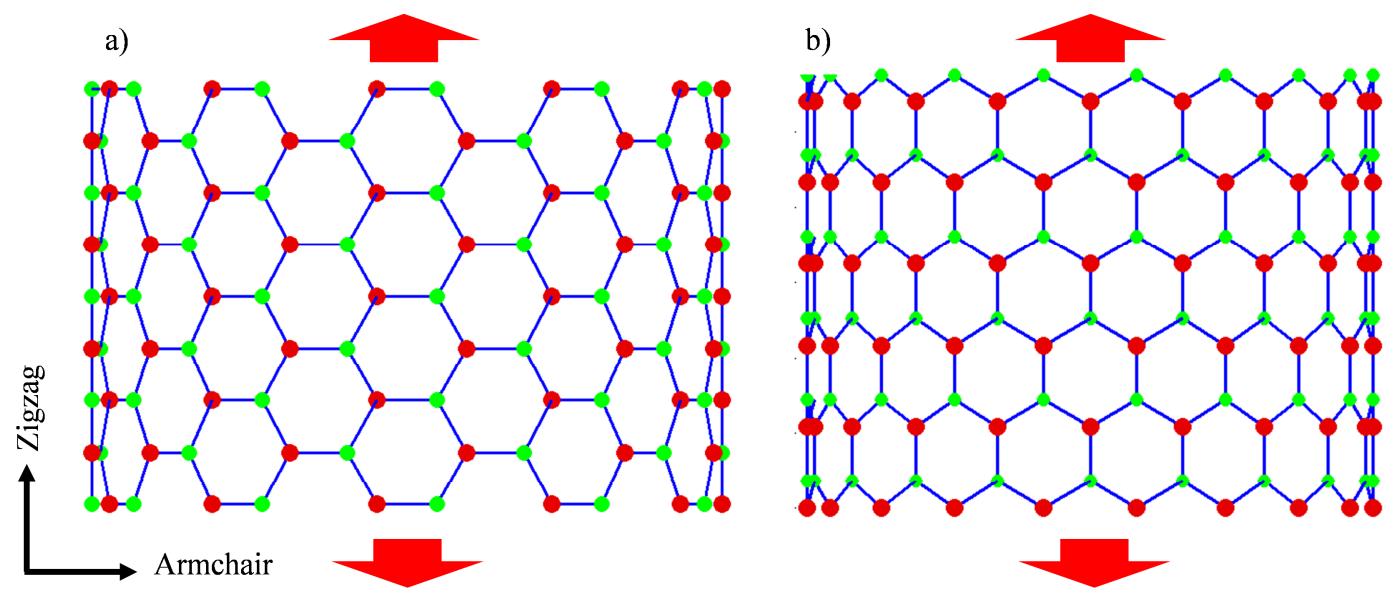

Figure 1. Schematic illustration of BN tubes: a) Armchair tube; b) Zigzag tube.

The arrows show the tensile direction.

\section{FRAMEWORK OF ANALYSIS}

Tersoff potential is used to model the interatomic interactions [32]. The potential energy $E$ of the atomic structure is a function of atomic coordinates as below:

$$
\begin{gathered}
E=\sum_{i} E_{i}=\frac{1}{2} \sum_{i \neq j} V_{i j}, V_{i j}=f_{C}\left(r_{i j}\right)\left[f_{R}\left(r_{i j}\right)+b_{i j} f_{A}\left(r_{i j}\right)\right] ; \\
f_{R}\left(r_{i j}\right)=A_{i j} \exp \left(-\lambda_{i j}^{I} r_{i j}\right), f_{A}\left(r_{i j}\right)=-B_{i j} \exp \left(-\lambda_{i j}^{I I} r_{i j}\right) ; \\
f_{C}\left(r_{i j}\right)= \begin{cases}1, & r_{i j} \leq R_{i j} ; \\
\frac{1}{2}+\frac{1}{2} \cos \left(\pi \cdot \frac{r_{i j}-R_{i j}}{S_{i j}-R_{i j}}\right), \quad R_{i j} \leq r_{i j} \leq S_{i j} ; \\
0, & r_{i j} \geq S_{i j} ;\end{cases} \\
b_{i j}=\chi_{i j}\left(1+\beta_{i}^{n_{i}} \zeta_{i j}^{n_{i}}\right)^{-1 / 2 n_{i}}, \zeta_{i j}=\sum_{k \neq i . j} f_{C}\left(r_{i k}\right) \omega_{i k} g\left(\theta_{i j k}\right), g\left(\theta_{i j k}\right)=1+c_{i}^{2} / d_{i}^{2}-c_{i}^{2} /\left[d_{i}^{2}+\left(h_{i}-\cos \theta_{i j k}\right)^{2}\right] \\
\lambda_{i j}^{I}=\left(\lambda_{i}^{I}+\lambda_{j}^{I}\right) / 2, \lambda_{i j}^{I I}=\left(\lambda_{i}^{I I}+\lambda_{j}^{I I}\right) / 2, A_{i j}=\sqrt{A_{i} A_{j}}, B_{i j}=\sqrt{B_{i} B_{j}}, R_{i j}=\sqrt{R_{i} R_{j}}, S_{i j}=\sqrt{S_{i} S_{j}} .
\end{gathered}
$$


where, the lower indices $i, j$ and $k$ label the atoms of the system, where interaction between atoms $i$ and $j$ is modified by a third atom $k . r_{i j}$ is the distance between atoms $i$ and $j ; f_{\mathrm{A}}$ and $f_{\mathrm{R}}$ are the attractive and repulsive pairwise terms; $f_{\mathrm{C}}$ is a cutoff function to ensure the nearest-neighbor interactions; $R_{i j}$ and $S_{i j}$ denote the small cutoff distance and the large one, respectively; $b_{i j}$ is a bond-order parameter, depending on the local coordination of atoms around atom $i$. Further detail of the Tersoff potential is available in [32]. Force field parameters are taken from the work by Sevik et al. [33] for B-N interactions.

Advanced computational techniques such as DFT calculations and MD simulations are time-consuming. Otherwise, atomic finite element method (AFEM) used both first and second derivatives of system energy in the energy minimization computation, hence it is faster than the standard conjugate gradient method which used only the first order derivative of system energy in MD simulation as discussed in [34]. AFEM, which has successfully analyzed the tensile response of various hexagonal sheets in our previous works [35 - 37], is used here. Further detailed numerical procedure of this method and our specific development for Tersoff potentials are available in our previous work [35] and references therein. We also follow here our previous scheme of the removal of the cutoff function, Eq. (1c), in Tersoff potential. This removal was explained in detail in [35]. Initial positions of atoms are generated by using the B-N bond length of $1.444 \AA$ taken from previous MD simulations [38] at optimized structure at $0 \mathrm{~K}$ with the same force field.

$\sigma$ and $\mathcal{E}$ denote nominal axial stress (engineering stress) and nominal axial strain (engineering strain), respectively. Young's modulus $Y$ is determined from the first derivative of the stress-strain curve at $\varepsilon=0$. Yt and $\sigma$ refer here as 2D Young's modulus (or in planestiffness) and 2D stress (or in-plane stress), respectively ( $t$ is the sheet's thickness). However, in the remainder of the paper we will use Young's modulus $Y t$ and axial stress $\sigma$ for short.

\section{RESULTS AND DISSCUSSION}

\subsection{Effects of the tubular length}

In this section, $(8,8) \mathrm{BN}$ armchair and $(14,0) \mathrm{BN}$ zigzag tubes having the same diameters are considered. This diameter is about 1.115 and $1.103 \mathrm{~nm}$ for $(8,8) \mathrm{BN}$ armchair and $(14,0)$ BN zigzag tubes, respectively. Difference in the diameter of these two tubes is about $1.1 \%$. Figure 2 shows the stress and Poisson's ratio-strain curves of $(8,8) \mathrm{BN}$ armchair and $(14,0) \mathrm{BN}$ zigzag tubes with various length-diameter ratios $L / D \sim 10,15,20$.

It can be seen from Fig. 2 a that stress-strain curves of $(14,0)$ zigzag tubes with various length-diameter ratios $\mathrm{L} / \mathrm{D} \sim 10,15,20$ are almost the same up to fracture points with maximum stress being $31.8 \mathrm{~N} / \mathrm{m}$ at strain $25.0-25.1 \%$ and then failure occurs absolutely at strain $25.5 \%$. This phenomenon also similarly occurs on $(8,8)$ armchair tubes with maximum stress being 37.2 $\mathrm{N} / \mathrm{m}$ at strain $29.2 \%$ and failure strain at about $30.3 \%$. In addition, axial stress in the armchair tube is higher than that in the zigzag one at the same axial strain. Thus, it can be said that performance of $(8,8)$ armchair tubes are better than $(14,0)$ zigzag ones under tension. We note that Anoop Krishnan and Ghosh [30] has also been reported similar result by using molecular dynamics simulations. 


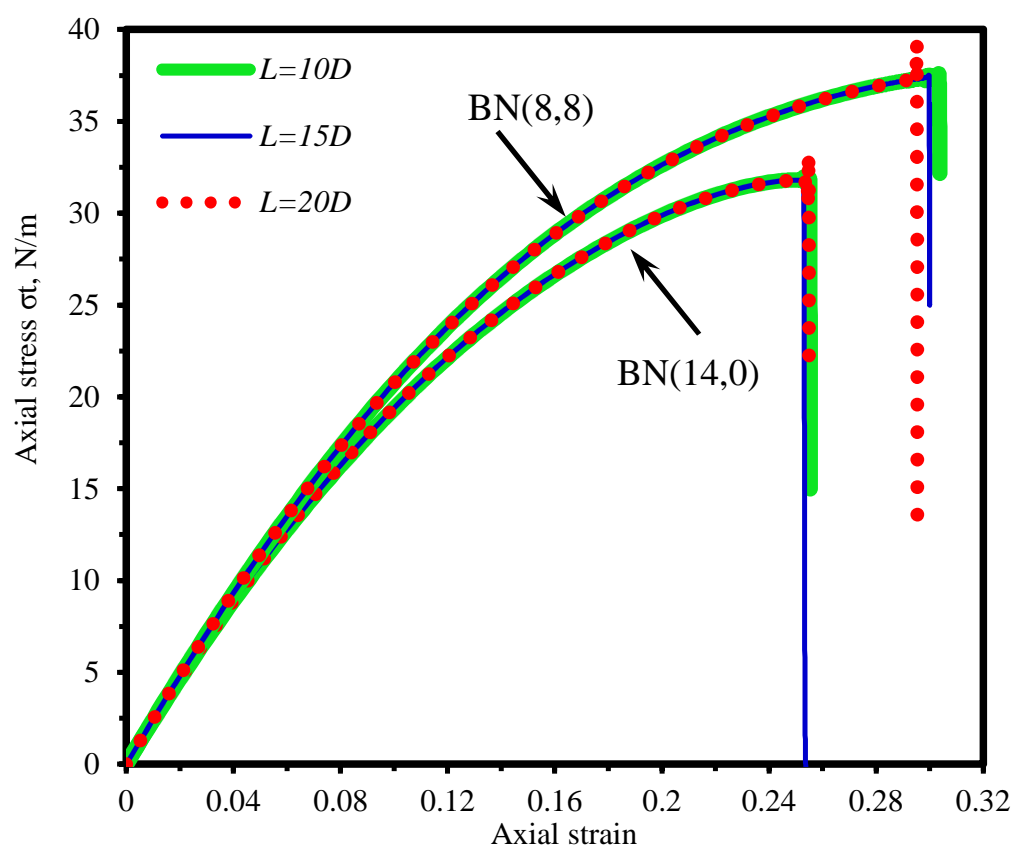

(a)

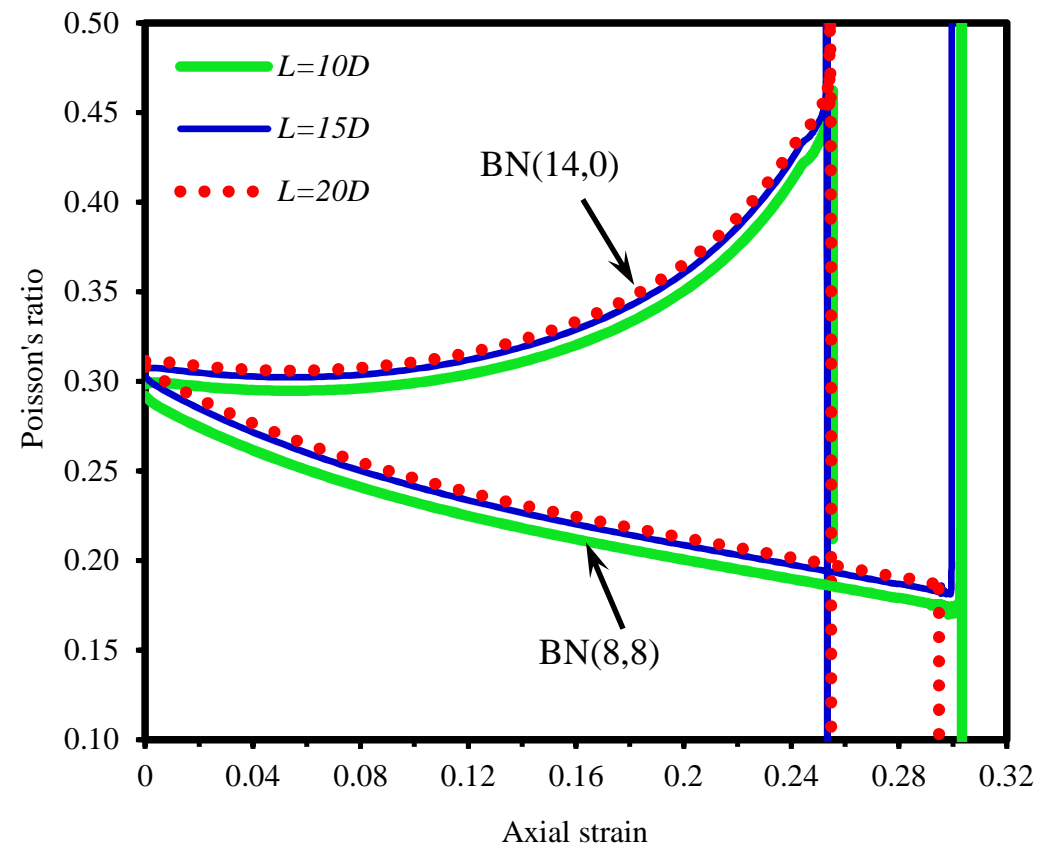

(b)

Figure 2. a) Stress-strain and b) Poisson's ratio-strain curves of $\mathrm{BN}(8,8)$ and $\mathrm{BN}(14,0)$ tubes with various length-diameter ratios $L / D$ being $10,15,20$.

Figure $2 \mathrm{~b}$ shows the effect of tubular length on Poisson's ratio of $(8,8) \mathrm{BN}$ armchair and $(14,0) \mathrm{BN}$ zigzag tubes. We can see that, at given strain, the Poisson's ratio of these tubes slightly increases when the length-diameter ratio increases. And Poisson's ratio of these tubes is approximate 0.3 at small strain. After that Poisson's ratio of $(8,8) \mathrm{BN}$ armchair tubes undergoes 
a significant reduction with increase axial strain while that of $\mathrm{BN}(14,0)$ tubes goes up. This phenomenon is probably due to armchair tubes having B-N bonds going around the tube and being parallel with the tangent direction (or perpendicular with tensile direction) while the ones in zigzag tubes make an angle of $30^{\circ}$ to the tangent direction (Figure 1). Thus, they resist deformation of tubular diameter in armchair tubes stronger than that in zigzag tubes under axial tension.

Table 1. Mechanics properties of $\mathrm{BN}(14,0)$ and $(8,8)$ under tension with various length-diameter ratios $L / D$ being $10,15,20$.

\begin{tabular}{lllllll}
\hline \hline $\begin{array}{l}\text { BN } \\
\text { tubes }\end{array}$ & $L / D$ & $\begin{array}{l}\text { Young's } \\
\text { modulus } Y t, \\
N / m\end{array}$ & $\begin{array}{l}\text { Maximum } \\
\text { stress } \sigma t, \\
N / m\end{array}$ & $\begin{array}{l}\text { Strain at } \\
\text { maximum } \\
\text { stress, } \%\end{array}$ & $\begin{array}{l}\text { Failure strain, } \\
\%\end{array}$ & Location of failure \\
\hline$(14,0)$ & 10 & 240.8 & 31.8 & 25.0 & 25.5 & $\begin{array}{l}\text { In the middle of tube } \\
\text { where undergo a } \\
\text { change of the diameter. }\end{array}$ \\
& 15 & 241.4 & 31.8 & 25.0 & 25.3 & \\
& 20 & 241.7 & 31.8 & 25.1 & 25.5 & At boundary \\
\hline$(8,8)$ & 10 & 246.0 & 37.2 & 29.2 & 30.3 & \\
& 15 & 246.1 & 37.2 & 29.2 & 30.0 & \\
& 20 & 246.1 & 37.2 & 29.2 & 29.5 & \\
\hline \hline
\end{tabular}

Table 1 shows that Young's modulus of both BN $(14,0)$ and BN $(8,8)$ almost remains unchanged when the tubular length increases with the former being about $241 \mathrm{~N} / \mathrm{m}$ and the latter being about $246 \mathrm{~N} / \mathrm{m}$. With assuming a nominal thickness of the sheet $t=3.35 \AA$, Young's modulus predicted by AFEM is about $719 \mathrm{GPa}$ for $\mathrm{BN}(14,0)$ and $734 \mathrm{GPa}$ for $\mathrm{BN}(8,8)$ tube, which is close to that of $722 \mathrm{GPa}$ using electric-field-induced resonance method [18].

It is found that these BN-NTs exhibit brittle fracture with a fast fracture process and a drop in its stress-strain curves (see Figure 2). In other words, buckling strains of these tubes (strain at maximum stress) are close to failure strain (see Table 1). For example, BN $(14,0)$ with $L=20 D$, we don't almost see the fracture at tensile strain $25.1 \%$ (Figure 3a), but it is buckling at tensile strain $25.4 \%$ (Figure 3b) and absolutely ruptured at tensile strain $25.5 \%$ (Figure 3c). Fracture took place at the middle of tube where underwent a change of the diameter. In contrast to, fracture of $\mathrm{BN}(8,8)$ armchair tubes occurs at boundary (see Figure 4) because its diameter remains unchanged during tension. This phenomenon has been explained above that $\mathrm{BN}(8,8)$ armchair tubes have B-N bonds going around the tube and being perpendicular with tensile direction which are resistant deformation in diameter direction.

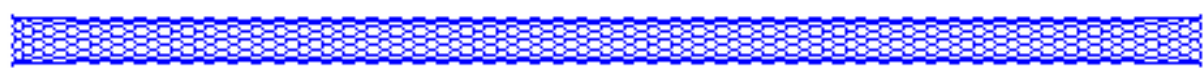

(a) $25.1 \%$

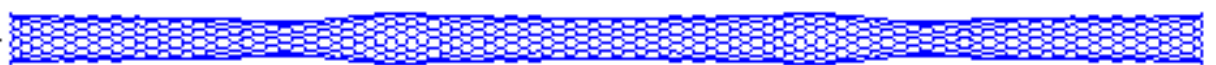

(b) $25.4 \%$
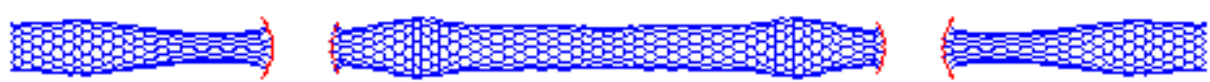

(c) $25.5 \%$

Figure 3. Snapshots of BN (14, 0), $L=20 \mathrm{D}$ under tensile strain: a) $25.1 \%$; b) $25.4 \%$; c) $25.5 \%$. 


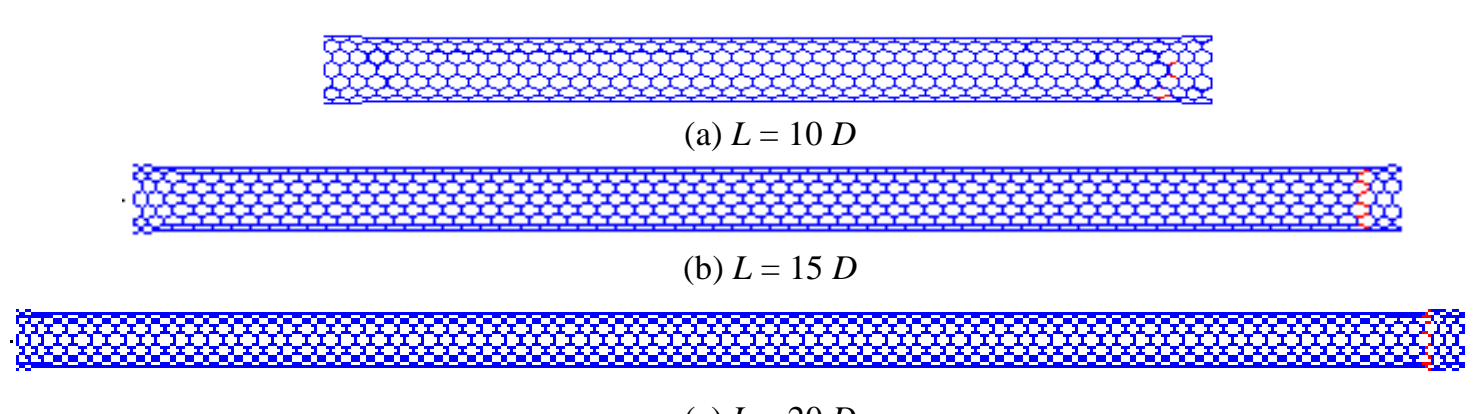

(c) $L=20 D$

Figure 4. Snapshots of $\mathrm{BN}(8,8)$ at fracture strain: a) $L=10 \mathrm{D}$; b) $L=15 \mathrm{D}$; c) $L=20 \mathrm{D}$.

\subsection{Effects of the tubular diameter}

For the purpose of investigating the effects of the tubular diameter's variations to mechanics behaviors of BN-NTs, we used four BN armchair tubes having the increase of diameter $(8,8),(10,10),(12,12)$, and $(15,15)$ and four BN zigzag tubes $(14,0),(17,0),(21,0)$, and $(25,0)$ having similar diameter, respectively. All above tubes have length-diameter ratio $\mathrm{L} / \mathrm{D} 10$. The result is listed in Table 2.

Table 2. Effect of tubular diameter on mechanical properties of BN-NTs under tension with length-diameter ratio L/D being about 10 .

\begin{tabular}{llllll}
\hline \hline BN tubes and sheets & $\begin{array}{l}\text { Diameter, } \\
\AA\end{array}$ & $\begin{array}{l}\text { Young's } \\
\text { modulus } Y t, \\
N / m\end{array}$ & $\begin{array}{l}\text { Maximum } \\
\text { stress } \sigma t, \\
N / m\end{array}$ & $\begin{array}{l}\text { Strain at } \\
\text { maximum } \\
\text { stress, } \%\end{array}$ & $\begin{array}{l}\text { Failure strain, } \\
\%\end{array}$ \\
\hline Zigzag sheet [35] & $\infty$ & 258 & 37.7 & 25.7 & - \\
$(15,15)$ armchair tube & 20.7 & 248 & 37.2 & 28.9 & 29.2 \\
$(12,12)$ armchair tube & 16.5 & 247 & 37.2 & 29.0 & 29.4 \\
$(10,10)$ armchair tube & 13.8 & 247 & 37.3 & 29.1 & 29.9 \\
$(8,8)$ armchair tube & 11.0 & 246 & 37.2 & 29.2 & 30.3 \\
\hline Armchair sheet [35] & $\infty$ & 251 & 35.5 & 26.4 & - \\
$(25,0)$ zigzag tube & 19.9 & 246 & 33.3 & 26.1 & 26.6 \\
$(21,0)$ zigzag tube & 16.7 & 245 & 33.0 & 25.9 & 26.8 \\
$(17,0)$ zigzag tube & 13.5 & 243 & 32.5 & 25.5 & 25.9 \\
$(14,0)$ zigzag tube & 11.1 & 241 & 31.8 & 25.0 & 25.5 \\
\hline \hline
\end{tabular}

It can be seen in Table 2 that the increase of diameter gives rise to the increase of Young's modulus of BN armchair (from 246 to $248 \mathrm{~N} / \mathrm{m}$ ) and zigzag tubes (from 241 to $246 \mathrm{~N} / \mathrm{m}$ ). At the same diameter, BN armchair tubes always have Young's modulus, maximum stress, failure strain higher than those of BN zigzag tubes.

Beside, strain at maximum stress of BN zigzag tubes slightly increases from $25.0 \%$ to 26.1 $\%$ when the tubular diameter is increased. In contrast to, that of BN armchair tubes gradually goes down from $29.2 \%$ to $28.9 \%$ with the rising of diameter. But the values of both BN zigzag 
and armchair tubes go close to that of armchair (26.4\%) and zigzag (25.7\%) sheet [35], respectively. It is easy to understand because a sheet can be looked like a tube with its diameter being infinite. It is also observed that the value of maximum stress of a $\mathrm{BN}$ armchair tube almost remains unchanged (about $37.2 \mathrm{~N} / \mathrm{m}$ ) irrespective of the tube diameter while that of BN zigzag tube slightly goes up from 31.8 to $33.3 \mathrm{~N} / \mathrm{m}$ with the increase of diameter. This result is similar to that reported in reference [30] using molecular dynamics simulation.

\section{CONCLUDING}

Mechanical properties of BN-NTs under uniaxial tension are investigated using atomic finite element method. Main findings are listed as follows:

- The BN armchair tubes exhibit stronger than the BN zigzag ones under axial tension.

- The BN-NTs exhibit brittle fracture. Location of fracture took place at the middle of BN $(14,0)$ zigzag tube where underwent a deformation of its diameter. In contrast to, fracture of $\mathrm{BN}(8,8)$ armchair tubes occurred at tensile boundary.

- Mechanical properties of BN-NTs are insignificantly dependent of the tubular length.

- Maximum stress of BN armchair tubes almost remains unchanged while that of BN zigzag tube slightly goes up when the tube diameter increases.

Acknowledgements. This research is funded by Vietnam National Foundation for Science and Technology Development (NAFOSTED) under grant number: 107.02-2016.13.

\section{REFERENCES}

1. Sazonova V., Yaish Y., Üstünel H., Roundy D., Arias T. A., and McEuen P. L. - A tunable carbon nanotube electromechanical oscillator, Nature 431 (7006) (2004) 284-287.

2. Moser J., Güttinger J., Eichler A., Esplandiu M. J., Liu D., Dykman M., and Bachtold A. Ultrasensitive force detection with a nanotube mechanical resonator, Nature nanotechnology 8 (7) (2013) 493-496.

3. Chaste J., Eichler A., Moser J., Ceballos G., Rurali R., and Bachtold A. - A nanomechanical mass sensor with yoctogram resolution, Nature Nanotechnology 7 (5) (2012) 301-304.

4. Biercuk M., Llaguno M. C., Radosavljevic M., Hyun J., Johnson A. T., and Fischer J. E. Carbon nanotube composites for thermal management, Applied Physics Letters 80 (15) (2002) 2767-2769.

5. Cadek M., Coleman J., Barron V., Hedicke K., and Blau W. - Morphological and mechanical properties of carbon-nanotube-reinforced semicrystalline and amorphous polymer composites, Applied Physics Letters 81 (27) (2002) 5123-5125.

6. Kim M., Park Y. B., Okoli O. I., and Zhang C. - Processing, characterization, and modeling of carbon nanotube-reinforced multiscale composites, Composites Science and Technology 69 (3) (2009) 335-342.

7. Iijima S. - Helical microtubules of graphitic carbon, Nature 354 (6348) (1991) 56-58.

8. Chopra N. G., Luyken R., Cherrey K., and Crespi V. H. - Boron nitride nanotubes, Science 269 (5226) (1995) 966. 
9. Loiseau A., Willaime F., Demoncy N., Hug G., and Pascard H. - Boron nitride nanotubes with reduced numbers of layers synthesized by arc discharge, Physical Review Letters 76 (25) (1996) 4737.

10. Narita I. and Oku T. - Synthesis of boron nitride nanotubes by using YB 6 powder, Solid State Communications 122 (9) (2002) 465-468.

11. Lourie O. R., Jones C. R., Bartlett B. M., Gibbons P. C., Ruoff R. S., and Buhro W. E. CVD growth of boron nitride nanotubes, Chemistry of Materials 12 (7) (2000) 1808-1810.

12. Wang J., Kayastha V. K., Yap Y. K., Fan Z., Lu J. G., Pan Z., Ivanov I. N., Puretzky A. A., and Geohegan D. B. - Low temperature growth of boron nitride nanotubes on substrates, Nano Letters 5 (12) (2005) 2528-2532.

13. Tang C., Ding X., Huang X., Gan Z., Qi S., Liu W., and Fan S. - Effective growth of boron nitride nanotubes, Chemical Physics Letters 356 (3) (2002) 254-258.

14. Pakdel A., Zhi C., Bando Y., Nakayama T., and Golberg D. - A comprehensive analysis of the CVD growth of boron nitride nanotubes, Nanotechnology 23 (21) (2012) 215601.

15. Golberg D., Bando Y., Eremets M., Takemura K., Kurashima K., and Yusa H. Nanotubes in boron nitride laser heated at high pressure, Applied Physics Letters 69 (14) (1996) 2045-2047.

16. Arenal R., Stephan O., Cochon J. L., and Loiseau A. - Root-growth mechanism for singlewalled boron nitride nanotubes in laser vaporization technique, Journal of the American Chemical Society 129 (51) (2007) 16183-16189.

17. Smith M. W., Jordan K. C., Park C., Kim J. W., Lillehei P. T., Crooks R., and Harrison J. S. - Very long single-and few-walled boron nitride nanotubes via the pressurized vapor/condenser method, Nanotechnology 20 (50) (2009) 505604.

18. Suryavanshi A. P., Yu M. F., Wen J., Tang C., and Bando Y. - Elastic modulus and resonance behavior of boron nitride nanotubes, Applied Physics Letters 84 (14) (2004) 2527-2529.

19. Golberg D., Costa P. M., Lourie O., Mitome M., Bai X., Kurashima K., Zhi C., Tang C., and Bando Y. - Direct force measurements and kinking under elastic deformation of individual multiwalled boron nitride nanotubes, Nano Letters 7 (7) (2007) 2146-2151.

20. Chopra N. G. and Zettl A. - Measurement of the elastic modulus of a multi-wall boron nitride nanotube, Solid State Communications 105 (5) (1998) 297-300.

21. Arenal R., Wang M. S., Xu Z., Loiseau A., and Golberg D. - Young modulus, mechanical and electrical properties of isolated individual and bundled single-walled boron nitride nanotubes, Nanotechnology 22 (26) (2011) 265704.

22. Chen Y., Zou J., Campbell S.J., and Le Caer G. - Boron nitride nanotubes: pronounced resistance to oxidation, Applied Physics Letters 84 (13) (2004) 2430-2432.

23. Baumeier B., Krüger P., and Pollmann J. - Structural, elastic, and electronic properties of $\mathrm{SiC}, \mathrm{BN}$, and $\mathrm{BeO}$ nanotubes, Physical Review B 76 (8) (2007) 085407.

24. Guo G., Ishibashi S., Tamura T., and Terakura K. - Static dielectric response and Born effective charge of BN nanotubes from ab initio finite electric field calculations, Physical Review B 75 (24) (2007) 245403. 
25. Chang C., Fennimore A., Afanasiev A., Okawa D., Ikuno T., Garcia H., Li D., Majumdar A., and Zettl A. - Isotope effect on the thermal conductivity of boron nitride nanotubes, Physical Review Letters 97 (8) (2006) 085901.

26. Meng W., Huang Y., Fu Y., Wang Z., and Zhi C. - Polymer composites of boron nitride nanotubes and nanosheets, Journal of Materials Chemistry C 2 (47) (2014) 10049-10061.

27. Nautiyal P., Gupta A., Seal S., Boesl B., and Agarwal A. - Reactive wetting and filling of boron nitride nanotubes by molten aluminum during equilibrium solidification, Acta Materialia 126 (2017) 124-131.

28. Attaccalite C., Wirtz L., Marini A., and Rubio A. - Efficient Gate-tunable light-emitting device made of defective boron nitride nanotubes: from ultraviolet to the visible, Scientific Reports 3 (2013).

29. Kang J. H., Sauti G., Park C., Yamakov V. I., Wise K. E., Lowther S. E., Fay C. C., Thibeault S. A., and Bryant R. G. - Multifunctional electroactive nanocomposites based on piezoelectric boron nitride nanotubes, Acs Nano 9 (12) (2015) 11942-11950.

30. Anoop Krishnan N. and Ghosh D. - Defect induced plasticity and failure mechanism of boron nitride nanotubes under tension, Journal of Applied Physics 116(4) (2014) 044313.

31. Anoop Krishnan N. and Ghosh D. - Chirality dependent elastic properties of single-walled boron nitride nanotubes under uniaxial and torsional loading, Journal of Applied Physics 115 (6) (2014) 064303.

32. Tersoff J. - Modeling solid-state chemistry: Interatomic potentials for multicomponent systems, Physical Review B 39 (8) (1989) 5566-5568.

33. Sevik C., Kinaci A., Haskins J.B., and Çağın T. - Characterization of thermal transport in low-dimensional boron nitride nanostructures, Physical Review B 84 (8) (2011) 085409.

34. Liu B., Huang Y., Jiang H., Qu S., and Hwang K. - The atomic-scale finite element method, Computer Methods in Applied Mechanics and Engineering 193 (17) (2004) 1849-1864.

35. Le M. Q. and Nguyen D. T. - Atomistic simulations of pristine and defective hexagonal $\mathrm{BN}$ and $\mathrm{SiC}$ sheets under uniaxial tension, Materials Science and Engineering: A 615 (2014) 481-488.

36. Le M. Q. and Nguyen D. T. - The role of defects in the tensile properties of silicene, Applied Physics A 118 (4) (2015) 1437-1445.

37. Nguyen D. T. and Le M. Q. - Mechanical properties of various two-dimensional silicon carbide sheets: An atomistic study, Superlattices and Microstructures 98 (2016) 102-115.

38. Le M. Q. - Atomistic study on the tensile properties of hexagonal AlN, BN, GaN, InN and $\mathrm{SiC}$ sheets, Journal of Computational and Theoretical Nanoscience 11 (6) (2014) 14581464. 